\title{
Producción de forraje verde hidropónico de maiz Zea mays. L. en invernadero con diferentes niveles de silicio
}

\author{
Evaluation of the production of green fodder hydroponically grown with different doses \\ of silicon from two varieties of maize Zea mays. L. under greenhouse conditions
}

\section{Elizabeth González M. ${ }^{1}$; Jesús Ceballos M. ${ }^{2}$; Orlando Benavides B. ${ }^{3}$}

1 Ingeniera Agrónoma, Universidad de Nariño. Pasto. Colombia.dianaeligm@hotmail.com

2 Estudiante de Pregrado I.A., Universidad de Nariño, Pasto.chucho-145@hotmail.com

3 Docente, I. A. M. Sc., Universidad de Nariño. Pasto,orlando.benavides2@gmail.com

Fecha recepción: Enero de 2015

Fecha de aceptación: Junio de 2015

\section{RESUMEN}

El objetivo de este trabajo fue evaluar la producción de forraje verde hidropónico en las variedades de maíz Morocho Blanco y Amarillo, sometidas a diferentes niveles de silicio bajo condiciones de invernadero ubicado en la granja experimental Botana de la Universidad de Nariño a $1^{\circ} 9^{\prime} 29.86^{\prime \prime}$ LN, 77²16 $33.85^{\prime \prime}$ LO, $2780 \mathrm{msnm}$. Se utilizó un experimento factorial de $2 \times 3$, donde un factor correspondió a las variedades de maíz y el otro a los niveles de silicio aplicados a la solución nutritiva $(0,0,025$ y $0,5 \mathrm{ml} / \mathrm{l})$ para un total de seis tratamientos y tres repeticiones. Las variables evaluadas fueron altura de plantas y rendimiento de forraje verde. En cuanto a altura de plantas el nivel de Silicio 0,025 ml/lobtuvo el mayor promedio $(14,48 \mathrm{~cm})$ en comparación con los niveles 0 y $0,5 \mathrm{ml} / 1$, los cuales no presentaron diferencias significativas entre ellos. En cuanto al rendimiento de forraje fresco, la interacción mostró que la variedad Morocho Blanco con la aplicación de $0,025 \mathrm{ml} / 1$ de silicio, estadísticamente fue mejor que las demás interacciones con promedio de $10,34 \mathrm{~kg} / \mathrm{m}^{2}$. Los demás tratamientos no presentaron diferencias significativas.

Palabras clave: Morocho blanco, morocho amarillo, silicio. 


\begin{abstract}
The objective of this study was to evaluate the production of hydroponic green fodder from Morocho Blanco and Morocho Amarillo maize varieties subjected to different doses of silicon under greenhouse conditions at the Botana experimental farm of the Universidad de Nariño $1^{\circ} 9^{\prime} 29.86^{\prime \prime} \mathrm{LN}, 7^{\circ} 16^{\prime} 33.85^{\prime \prime} \mathrm{LO}, 2870 \mathrm{msnm}$. A $2 \times 3$ factorial design was applied, where one factor corresponded to the maize varieties and the other factor to the silicon doses $(0,025$ and $0,5 \mathrm{ml} / \mathrm{L})$ that were applied to the nutrient solution, for a total of six treatments and three repetitions. The variables that were evaluated were plant height and green fodder yield. Regarding plant height, the silicon dose of $0,025 \mathrm{ml} / \mathrm{L}$ showed the highest average $(14,48 \mathrm{~cm})$ compared to 0 and $0,5 \mathrm{ml} / \mathrm{L}$ doses, which did not show significant differences among them. In terms of fresh fodder yield, the interaction showed that the Morocho Blanco variety with a silicon application of 0,025 $\mathrm{mg} / \mathrm{L}$ was statistically better than the other interactions, with an average of $10,34 \mathrm{Kg} / \mathrm{m}^{2}$. The other treatments did not show significant differences.
\end{abstract}

Keywords: Morocho blanco, morocho amarillo, silicone.

\section{INTRODUCCIÓN}

El departamento de Nariño se caracteriza por tener ecosistemas aptos para sistema lecheros y especialmente para especies menores como el cuy Cavia porcellus L. Cerca del $80 \%$ de las familias que viven en la zona rural mantienen pequeños predios con estas especies. Sin embargo, la alimentación utilizada para estos animales presenta en la actualidad inconvenientes derivados de los efectos negativos del cambio climático y de los requerimientos agronómicos para la producción de forraje (Agronet, 2009).

Lo anterior reviste gran importancia, ya que es preocupación de los ganaderos y productores de especies menores, dada la creciente variabilidad del clima y las posibles contingencias adversas como ataque de plagas y enfermedades e incertidumbre por situaciones negativas que pudiesen representar disminución en los ingresos familiares y deterioro de la producción (Espinoza et al., 2004).

Las variaciones climáticas junto con la baja calidad de los forrajes utilizados en la producción pecuaria, constituyen factores que restringen el desarrollo adecuado de la ganadería nacional. Por ello, los productores agropecuarios suministran a sus animales, dietas suplementarias basadas en alimentos concentrados (Espinoza et al., 2004), las cuales tienen alto costo, debido a que los insumos para su elaboración son importados.

El cultivo hidropónico de plantas con fines forrajeros (maíz, cebada, avena, sorgo, alfalfa, etc.), puede resultar provechoso en la alimentación animal, permitiendo cultivar especies altamente productivas en medios artificiales o sustratos, en donde las raíces se desarrollan adecuadamente (Durany, 1984; Urias, 1997). 
La producción de forraje bajo sistemas tradicionales implica trabajos de adecuación que comprenden preparación de terreno, manejo agronómico del cultivo y obtención de cosechas de forraje que superan en tiempo más de 60 días; además de que la producción por metro cuadrado es mucho menor que en condiciones hidropónicas. Por otra parte para obtener entre 1 a $8 \mathrm{~kg}$ de materia seca de alimento utilizando cultivos en suelo se emplea un metro cúbico de agua, mientras que ese mismo volumen de agua en forraje verde hidropónico se obtienen alrededor de $100 \mathrm{~kg}$ (FAO, 2006).

La producción de forraje verde hidropónico (FVH) consiste en la germinación de semillas de gramíneas o leguminosas y posterior crecimiento bajo condiciones ambientales controladas (luz, temperatura y humedad) en ausencia de suelo (FAO, 2002). Su uso se destina para la alimentación de bovinos, ovinos, caprinos, equinos, porcinos, conejos y aves (Müller et al., 2005a; Herrera et al., 2007).

El FVH ofrece una serie de ventajas, como producción forrajera durante todo el año, desarrollo del cultivo en pequeñas áreas, aporte de complejos vitamínicos necesarios, no ocasionan trastornos digestivos y rápida recuperación de la inversión (FAO, 2002; Müller et al., 2005b).

Los fenómenos climatológicos, tales como sequías prolongadas, nevadas, inundaciones y las lluvias de cenizas volcánicas, han venido incrementando significativamente su frecuencia en estos últimos años, afectando negativamente la producción o limitando el acceso al forraje producido en forma convencional para la alimentación de los animales. Ello redunda en la necesidad de contar con alternativas de producción de forraje que permitan prevenir pérdidas productivas especialmente a nivel de los pequeños y medianos productores ganaderos o de especies menores (FAO, 2006). Frente a estas circunstancias de déficit alimentario, surge como una alternativa importante, la implementación de un sistema de producción de FVH.

El FVH efectuado a partir de semillas de maíz, cebada, trigo, entre otros, aporta en términos generales mayor energía, proteína y digestibilidad (FAO, 2006); es una alternativa de producción para los ganaderos y productores de Nariño y del País para mejorar la alimentación animal, además de ofrecer la ventaja en cuanto al aprovechamiento de toda la planta.

Una de las plantas más utilizadas con fines forrajeros ha sido el maíz (Zea mays L.) por su elevado valor nutritivo y altos rendimientos lo cual permite que en diversos medios de producción hidropónicos, se generen elevados y constantes volúmenes de FVH produciendo alimento a menor costo que el sistema convencional de producción de forrajes a campo abierto (FAO, 2006).

Por otro lado, los fertilizantes con silicio asimilable, se presentan como alternativa para mitigar el impacto de los factores que contribuyen a la desertificación y contribuyen con la sostenibilidad de la agricultura intensiva en zonas áridas y semiáridas, así como en cultivos hidropónicos (Quero, 2007).

Además de lo anterior, con la aplicación de silicio hay un doble efecto en el sistema 
suelo-planta. Primeramente, refuerza en la planta su capacidad de almacenamiento $y$ distribución de carbohidratos requeridos para el crecimiento y producción, la autoprotección contra enfermedades causadas por hongos y bacterias, el ataque de insectos y ácaros y de las condiciones desfavorables de clima, al estimular eldesarrolloy actividad deestructuras poliméricas en cutículas, los tricomas y fitolitos en la superficie de las hojas. En segundo lugar, la retención y disponibilidad del agua, sus propiedades físicas y químicas y de mantener los nutrientes en forma disponible para la planta. La aplicación de fertilizantes minerales con silicio es una alternativa que se puede considerar bajo un esquema de agricultura sustentable y altamente efectiva en cualquier tipo de suelo, sobre todo en aquellos donde el pH es inferior a 6,0 (Rivera et al., 2010).

Acorde con lo anterior, el objetivo de este trabajo fue evaluar el efecto de diferentes niveles de silicio en soluciones hidropónicas sobre los componentes forrajeros de dos variedades regionales de maíz Zea mays. L. (Morocho Blanco y Morocho Amarillo).

\section{MATERIALES Y MÉTODOS}

Localización. El presente trabajo se realizo bajo condiciones de invernadero ubicado en la granja experimental Botana de la Universidad de Nariño a $1^{\circ} 9^{\prime} 29.86^{\prime \prime} \mathrm{LN}, 77^{\circ} 16^{\prime} 33.85^{\prime \prime} \mathrm{LO}, 2780$ msnm, con temperatura promedio anual de $13^{\circ} \mathrm{C}$, precipitación media anual de $910,3 \mathrm{~mm}$ y humedad relativa de 75\% (IDEAM, 2010).
Contenedores. Se instalaron dentro del invernadero dos módulos hidropónicos en ángulo de aluminio, a $30 \mathrm{~cm}$ del piso, altura de $180 \mathrm{~cm}$. Cada módulo presentaba tres niveles separados a un espacio vertical de $80 \mathrm{~cm}$ y pendiente de $1 \%$. Los módulos sirvieron de soporte para las bandejas donde se sembraron las semillas de maíz. Estos módulos se cubrieron con polietileno negro calibre 2 durante el periodo de germinación de las semillas para brindar condiciones de oscuridad y temperatura favorables para la germinación. Los contenedores correspondieron a bandejas de asbesto cemento, con dimensiones de 0,60 m de ancho por 1,60 m de largo y $3 \mathrm{~cm}$ de profundidad, en las cuales se depositaron las semillas sirviendo como cama para la misma y posterior producción del forraje.

Riego. El sistema de riego fue por nebulización. Las líneas de microaspersores se colocaron a una altura de 0,40 m de las bandejas de cultivo.

Diseño experimental. Se utilizó un experimento factorial de $2 \times 3$, donde un factor correspondió a las variedades de maíz Morocho Amarillo y Morocho Blanco. El otro factor fue Silicio con tres niveles aplicados a la solución nutritiva $(0,0,025$ y $0,5 \mathrm{ml} / \mathrm{l}$ ) para un total de seis tratamientos $\mathrm{y}$ tres repeticiones.

Material vegetal. Morocho Amarillo y Morocho Blanco son dos variedades regionales de maíz de uso común por los ganaderos del departamento de Nariño, consideradas de buena producción de forraje. En el ensayo se utilizó un total de $54 \mathrm{~kg}$ de semilla para las 18 bandejas. Por cada bandeja se sembraron $3 \mathrm{~kg}$ de semilla. 
Preparación de la semilla. Para suspender la latencia de las semillas, éstas se embebieron en agua durante 24 horas según lo indicado por Quispe (2011).

Siembra. Cumplido el tiempo de imbibición se depositaron las semillas homogéneamente en las bandejas tapándose posteriormente con plástico negro. Cuando se logró el 95\% de germinación se retiró el plástico y quedaron expuestas las semillas a condiciones de humedad y temperatura adecuadas para favorecer el crecimiento y desarrollo de las plántulas de cada bandeja.

Solución nutritiva. La solución nutritiva empleada correspondió a una formula soluble NPK (25-5-5), la cual fue enriquecida con un fertilizante (SIFOL) a base de fosfitos de potasio, calcio, cobre y una alta concentración de Silicio soluble $\left(\mathrm{S}_{\mathrm{i}} \mathrm{O}_{2} ; 200 \mathrm{~g} / \mathrm{l}\right)$. Las dosis de fertilización planteadas se aplicaron dejando un día por medio y las concentraciones de silicio fueron aplicadas a los 10 y 18 días después de la germinación.

\section{Variables Evaluadas}

Altura de plantas y producción de forraje fresco. La altura y el rendimiento de forraje fresco fueron medidos al momento de la cosecha (25 días después de germinación) en cada una de las unidades experimentales, teniendo en cuenta que este es el tiempo total de producción del cultivo para obtener el forraje verde hidropónico.

Análisis estadístico. Los datos obtenidos fueron sometidos al Análisis de Varianza (ANDEVA) y prueba de comparación de medias de Tukey $(\mathrm{P} \leq 0,05)$.

\section{RESULTADOS Y DISCUSION}

Altura de planta (AP). En AP, se observaron diferencias estadísticas significativas entre niveles de Silicio (Si) (Tabla 1). Con respecto a la variedad y a la interacción variedad por nivel de silicio (VAR x Si) no se presentaron diferencias estadísticas en cuanto a altura de planta.

Tabla 1. ANDEVA para la altura de planta en $\mathrm{cm}$, con diferentes niveles de Silicio aplicados a las variedades de maíz Morocho Blanco y Morocho Amarillo, bajo condiciones hidropónicas.

\begin{tabular}{lccccc}
\hline \multicolumn{1}{c}{ FV } & SC & GL & CM & F cal & p-valor \\
\hline Modelo & 89,10 & 5 & 17,82 & 4,51 & 0,0152 \\
Variedad (VAR) & 9,83 & 1 & 9,83 & 2,49 & 0,1407 \\
Silicio (Si) & 71,21 & 2 & 35,61 & 9,01 & 0,0041 \\
VAR x Si & 8,06 & 2 & 4,03 & 1,02 & 0,3899 \\
\hline Error & 47,41 & 12 & 3,95 & & \\
\hline
\end{tabular}

Diferencias significativas $=\mathrm{p}<0,05 ; \mathrm{F}$ cal $=\mathrm{F}$ calculado. 
De acuerdo con los resultados obtenidos para esta variable, el nivel de Si 0,025 ml presentó el mejor promedio en comparación con los niveles de silicio 0 y $0,5 \mathrm{ml}$ los cuales no fueron estadísticamente iguales entre sí (Tabla 2).

Tabla 2. Altura de planta en cm de maíz bajo condiciones hidroponicas e invernadero bajo tres niveles de aplicación de Silicio.

\begin{tabular}{ll}
\hline Nivel de Silicio $(\mathrm{ml} / \mathrm{l})$ & Medias \\
\hline Silicio 0,025 & $14,48 \mathrm{a}$ \\
Silicio 0 & $10,52 \mathrm{~b}$ \\
Silicio 0,5 & $10,05 \mathrm{~b}$ \\
\hline
\end{tabular}

Medias con una letra común no son significativamente diferentes $(p>0,05)$.

Lo anterior se puede explicar en términos de que algunas especies vegetales como la calabaza y el arroz acumulan concentraciones apreciables de Si en sus tejidos y mejoran su crecimiento y fertilidad cuando se les suministra cantidades adecuadas de dicho elemento (Horna, 2007). El Si se deposita en forma amorfa en las paredes celulares. Contribuye con las propiedades mecánicas de la pared como son la rigidez y la elasticidad. En las gramíneas, no solamente se deposita en la pared celular de la epidermis, pelos, brácteas, entre otros, sino también en el interior, como sucede en las células buliformes y en el xilema.

Según Reina (2010) la presencia de Si en las plantas hace que en las hojas y tallos, se incremente la cantidad de oxígeno que expulsan las plantas hacia la raíz llegando al parénquima, oxidando de ésta manera, la rizosfera y haciendo que el Fe y Mn reducido se oxide. De esta forma, se evita una toma excesiva de éstos elementos, que pueden llegar a ser tóxicos para la planta. El Si refuerza en la planta su capacidad de distribución de carbohidratos requeridos para el crecimiento y producción de cosecha, tiene acción sinérgica con el Calcio, el Magnesio y el Potasio, mejorando la vida media de las cosechas perecederas, incrementando la eficiencia de las prácticas de post-cosecha (Quero y Cárdenas, 2007).

En las gramíneas, el Si se acumula en cantidades mayores que cualquier otro elemento inorgánico (Agnusdey, 2007), excepto en ciertas algas, diatomeas y equisetáceas (cola de caballo), donde no es considerado un elemento esencial para estas plantas. Como resultado de esto, el Si es omitido en la formulación de soluciones de cultivo de uso rutinario y no es considerado en muchas investigaciones de fisiología vegetal. Sin embargo, las evidencias muestran que las estructuras de las plantas que crecen en ausencia de $\mathrm{Si}$, frecuentemente son más débiles y su crecimiento, desarrollo, viabilidad y reproducción son anormales, son más susceptibles al estrés abiótico, como toxicidad por metales, fácilmente invadidas por organismos patógenos, insectos fitófagos y mamíferos herbívoros. La mayoría de estas respuestas se observan en plantas que crecen en suelos pobres en Si (Epstein, 1994).

Forraje fresco hidropónico. Para el rendimiento de forraje fresco hidropónico, se encontraron significancias para niveles de Si y la interacción variedad por nivel de silicio (VAR x Si). Con respecto a la variedad, no se presentaron diferencias significativas (Tabla 3 ). 
Tabla 3. ANDEVA para la producción de forraje verde hidropónico con diferentes niveles de Silicio aplicados a las variedades de maíz Morocho Blanco y Morocho Amarillo, bajo condiciones hidropónicas.

\begin{tabular}{lccccc}
\hline FV & SC & GL & CM & F & p-valor \\
\hline Modelo & 23,27 & 5 & 4,65 & 5,50 & 0,0074 \\
Variedad (VAR) & 3,04 & 1 & 3,04 & 3,59 & 0,0824 \\
Silicio (Si) & 12,84 & 2 & 6,42 & 7,58 & 0,0074 \\
VAR x Si & 7,39 & 2 & 3,69 & 4,36 & 0,0377 \\
\hline Error & 10,16 & 12 & 0,85 & & \\
\hline
\end{tabular}

Diferencias significativas $=p<0,05$

La interacción variedad por nivel de Si (Tabla 4) mostró que la variedad Morocho Blanco con la aplicación de 0,025 ml/1 de Si, estadísticamente fue mejor que las demás interacciones con un promedio de rendimiento de forraje fresco de 10,34 $\mathrm{kg} / \mathrm{m}^{2}$. Los demás tratamientos no presentaron diferencias estadísticas entre sus promedios.

Tabla 4. Comparación de medias de la producción de forraje verde hidropónico en la interacción variedades por niveles de Silicio.

\begin{tabular}{lcc}
\hline Variedad & $\begin{array}{c}\text { Nivel de } \\
\mathrm{Si}(\mathrm{ml} / \mathrm{l})\end{array}$ & $\begin{array}{c}\text { Rendimiento } \\
\left(\mathrm{kg} / \mathrm{m}^{2}\right)\end{array}$ \\
\hline Morocho Blanco & 0,025 & $10,34 \mathrm{a}$ \\
Morocho Amarillo & 0,025 & $7,60 \mathrm{~b}$ \\
Morocho Amarillo & 0 & $7,19 \mathrm{~b}$ \\
Morocho Amarillo & 0,5 & $7,11 \mathrm{~b}$ \\
Morocho Blanco & 0,5 & $7,08 \mathrm{~b}$ \\
Morocho Blanco & 0 & $7,05 \mathrm{~b}$ \\
\hline
\end{tabular}

Medias con una letra común no son significativamente diferentes (Tukey, $\mathrm{P} \leq 0,05$ )

El hecho de no encontrar demasiadas diferencias en las medias de tratamiento, corrobora el planteamiento que el Si no está considerado en la agricultura como un microelemento esencial en el desarrollo de las plantas cultivadas; Sin embargo, (Reina, 2010) indica que las principales gramíneas (arroz, caña de azúcar, sorgo, maíz, trigo, millo, avena), forrajeras (kikuyo, bermuda, alfalfa), frutales (mango, aguacate, plátano, banano, feijoa), y otras como tomate, pepino, algodón, fríjol y crucíferas, responden con mayor productividad y sanidad, cuando hay concentraciones de Si disponibles en el suelo.

\section{CONCLUSIONES}

Con la aplicación de 0,025 ml/1 en condiciones hidropónicas, se obtuvo la mayor altura de planta de maíz. En el caso de las interacciones, el mejor rendimiento de forraje verde hidropónico, se obtuvo con la aplicación de $0,025 \mathrm{ml} / 1$ en la variedad Morocho Blanco.

\section{REFERENCIAS}

AGNUSDEY, M. 2007. Calidad nutritiva del forraje. Grupo de producción y utilización de pasturas. Argentina. En: http://www.produccion-animal.com. ar; consulta: febrero, 2014. 
AGRONET (RED DE INFORMACIÓN Y COMUNICACIÓN ESTRATÉGICA DEL SECTOR AGROPECUARIO). 2009. Sistema de información de precios e insumos y factores asociados a la producción. En: http: agronet.gov.co/; consulta: noviembre, 2013.

CÁZAREZ, J. 2010. Con silicio, hasta 24 toneladas de maíz por hectárea. El Diario. Los Mochis México. En: http://www.eldiariodelosmochis.com.mx/movil / publicacion.php?id=50256; consulta: noviembre, 2013.

DURANY, U. 1984. Hidroponía. Cultivo de Plantas sin tierra. 5taEd. Editorial Sintes, S.A, Barcelona, España.61p.

EPSTEIN, E. 1994. The anomaly of silicon in plant biology. Proceedings of the National Academy of Sciences. USA. 91:11 - 17

ESPINOZA, F., ARGENTI, G., URDANETA, C., ARAQUE, A., FUENTES, J., PALMA, C., Y BELLO, C. 2004. Uso del forraje de maíz Zea mays L. hidropónico en la alimentación de toretes mestizos. Zootecnia Tropical. 22(4):303 - 315.

FAO y CIMMYT, 1997.El maíz blanco Un cereal de consumo humano tradicional en los países en desarrollo. En: ftp://ftp.fao.org/docrep/fao/006/ w2698s/w2698s00.pdf; consulta: octubre, 2013.

ORGANIZACIÓN DE LAS NACIONES UNIDAS PARA LA ALIMENTACIÓN Y LA AGRÍCULTURA. (FAO). 2006. Manual técnico forraje verde hidropónico. Oficina regional de la FAO para America Latina y el Caribe. Santiago- Chile. En: http:/ / www.fao.or g/ docrep/field/009/ah472s/ ah472s00.htm; consulta: septiembre, 2013.

ORGANIZACIÓN DE LAS NACIONES UNIDAS PARA LA ALIMENTACIÓN Y LA AGRÍCULTURA. FAO. 2002. Manual Técnico: Forraje Verde Hidropónico. Oficina Regional de la FAO para América Latina y El Caribe. Santiago de Chile, Chile.
HERRERA, A., DEPABLOS, R., LÓPEZ, M., BENEZRRA, G., y RÍOS, L. 2007. Degradabilidad y digestibilidad de la materia seca del forraje hidropónico de maíz (Zea mays L.). Respuesta animal en términos de consumo y ganancia de peso. Revista Científica, XVII (4):372 - 379.

HORNA, R. 2007. Efectos del silicio en la nutrición vegetal- producción de silicio orgánico. Agripitus. Quevedo Ecuador. En: http://www.uteq.edu.ec/ eventos/2007/congreso_biotecnologia/biotecnologia/archivos/832.pdf; consulta: noviembre, 2013.

INSTITUTO DE HIDROLOGÍA, METEOROLOGÍA Y ESTUDIOS AMBIENTALES (IDEAM). 2010. Boletín divulgativo de información climatológica anual. Pasto Nariño. Consulta: noviembre, 2013.

MÜLLER, L., MANFRON, V. SANTOS, S. MEDEIROS, V. HAUT, D. DOURADO, E. BINOTTO, E., y. BANDEIRA, A. 2005a. Produção e composição bromatologica da forragem hidropônica de milho, Zea mays L., com diferentes densidades de semeadura e datas de colheita. Zootecnia Tropical. 23(2):105 - 119.

MÜLLER, L., SANTOS, S. MANFRON, V. HAUT, D. BINOTTO, S. MEDEIROS, V. y DOURADO, E. 2005b. Produção e qualidade bromatológica de gramíneas em sistema hidropônico. Uruguaiana, Revista da FZVA. 12(1):88 - 97.

PIRELA, M. 2005. Valor nutritivo de los pastos tropicales. Instituto nacional de investigaciones agrícolas. En: http://www.avpa.ula.ve; consulta: febrero, 2014.

QUERO, G., y CARDENAS V. 2007 Nueva tecnología para incrementar la producción agrícola tecnificada y tradicional y mejorar la calidad agrícola de los suelos, Instituto Tecnológico Superior de Uruapan. Michoacán, México. (201-205) 
QUERO, G. 2007. Fertilización para el crecimiento generativo en cultivos bajo invernadero. En: http:/ / assets00.grou.ps/0F2E3C/wysiwyg_files/FilesModule/ingenieriaagronomica/20101023152657-zobjbencyianbldyg/FERTILIZACION_BAJO_EL_CRECIMIENTO_GERMINATIVO_BAJO_INVERNADERO.pdf; consulta: noviembre, 2013.

QUISPE, A.2011. Forraje verde hidropónico. Centro de investigación de hidroponía y nutrición mineral. Universidad Nacional Agraria La Molina. Perú. En: http://www. Lanolina.edu.pe. Consulta: noviembre, 2013.

REINA, H. 2010. Importancia del silicio en suelo y plantas. En: http:/ / www.freshplaza.es/news_detail. asp?id=37011; consulta: noviembre, 2013.

RIVERA, A., MORONTA, M., GONZÁLEZ, M., GONZÁlEZ, D., PERDOMO, D., GARCÍA, D., Y HERNÁNDEZ, G. 2010. Producción de forraje verde hidropónico de maíz (Zea mays L.) en condiciones de iluminación deficiente. Zootecnia Tropical. 28(1):33 - 41 .

URIAS, E. 1997. Hidroponía. Como cultivar sin tierra. Red de Hidroponía. Lima, Perú. 7p. 